\title{
Associations between chronic disease, age and physical and mental health status
}

\author{
W. M. Hopman, MA (1,2); M. B. Harrison, PhD (2,3,4); H. Coo, MSc (2); E. Friedberg, MHA (3,4); M. Buchanan, BScN (3);
}

E. G. VanDenKerkhof, DrPH $(2,3,5)$

\begin{abstract}
This paper examines the associations between chronic disease, age, and physical and mental health-related quality of life (HRQOL), using data collected in 10 studies representing five chronic conditions. HRQOL was measured using the SF-36 or the shorter subset, SF-12. Physical Component Summary (PCS) and Mental Component Summary (MCS) scores were graphed by condition in age increments of 10 years, and compared to age- and sex-adjusted normative data. Linear regression models for the PCS and MCS were controlled for available confounders. The sample size of 2418 participants included 129 with renal failure, 366 with osteoarthritis (OA), 487 with heart failure, 1160 with chronic wound (leg ulcer) and 276 with multiple sclerosis (MS). For the PCS, there were large differences between the normative data and the mean scores of those with chronic diseases, but small differences for the MCS. Female gender and comorbid conditions were associated with poorer HRQOL; increased age was associated with poorer PCS and better MCS. This study provided additional evidence that, while physical function could be severely and negatively affected by both chronic disease and advanced age, mental health remained relatively high and stable.
\end{abstract}

Key words: age, chronic disease, mental health, physical health, HRQOL, status, SF-36, SF-12

\section{Background}

Health-related quality of life (HRQOL) is a primary concern with chronic conditions and is often used as a research outcome in both clinical trials and observational studies. A useful characteristic of a generic measure of HRQOL is the ability to compare across different diseases to assess burden of illness. ${ }^{1}$ HRQOL is a particularly relevant outcome in chronic disease, where a cure is often unavailable and health goals involve living with and managing one's condition. ${ }^{2,3}$

A growing body of research has examined HRQOL in a variety of diseases, and many studies have identified significant impairments. $^{4-10}$ HRQOL has also been examined in the general population, providing normative data for comparative purposes. ${ }^{4-6,11}$ However, while chronic disease typically has a significant negative impact on the physical aspects of health, mental health status may remain relatively unaffected. This has been demonstrated in studies on individual conditions, ${ }^{12-17}$ as well as in two multiple-condition studies: one focusing on allergies, arthritis, congestive heart failure, chronic lung disease, hypertension, diabetes and ischemic heart disease, ${ }^{1}$ and another that compared multiple sclerosis (MS), osteoarthritis (OA), renal disease and renal transplants. ${ }^{2}$ Additional research into the effect of multimorbidity, taking into account the effect of both the number and severity of comorbid conditions, also identified an inverse association between the number and severity of conditions and HRQOL, ${ }^{18,19}$ particularly in the physical domains. ${ }^{18,19}$

Cross-sectional evidence suggests that while the physical aspects of HRQOL decline as age increases, ${ }^{2,4}$ mental health remains stable across age categories, or may even improve., ${ }^{2,421}$ This observation is further supported with longitudinal data. HRQOL tends to be stable over three ${ }^{22}$ to five $^{23}$ years, but if there are changes, it is the physical aspects of HRQOL which tend to decline while mental aspects improve. ${ }^{23}$

The purpose of this study was to examine the relationship between age and physical and mental aspects of health for people with different chronic conditions. Research objectives included a comparison of the physical and mental health status across diseases, as well as an examination of the association between age and HRQOL, while controlling for key variables available across all databases. We hypothesized that the physical aspects of HRQOL would be substantially lower in those with chronic disease as compared to a normative population, and that it would also be lower in older versus younger age groups, while the mental aspects of HRQOL would be similar to the normative data and be relatively unaffected by disease group or increased age.

Herein, we examined data collected in ten Canadian studies representing five conditions, including renal failure, hip and knee OA, congestive heart failure ( $\mathrm{CHF}$,

Author References

1 Clinical Research Centre, Kingston General Hospital, Kingston, ON.

2 Department of Community Health and Epidemiology, Queen's University, Kingston, ON.

3 School of Nursing, Faculty of Health Sciences, Queen's University.

4 Ottawa Health Research Institute, Clinical Epidemiology Program, Ottawa, ON.

5 Department of Anesthesiology, Faculty of Health Sciences, Queen's University.

Correspondence: Wilma M. Hopman, MA, Clinical Research Centre, Angada 4, Kingston General Hospital, Kingston, ON, Canada K7L 2V7,

Tel.: 613-549-6666, ext. 4941, Fax: 613-548-2428, Email: hopmanw@kgh.kari.net 
TABLE 1

Characteristics of the ten studies

\begin{tabular}{|c|c|c|c|c|c|c|}
\hline Study & $\mathbf{n}$ & Inclusion criteria & Exclusion criteria & Age Mean (SD) & PCS Mean (SD) & MCS Mean (SD) \\
\hline Renal failure & 129 & $\begin{array}{l}\text { Age } 18 \text { years } \\
>6 \text { months duration }\end{array}$ & $\begin{array}{l}\text { Acute/reversible } \\
\text { cognitive impairment }\end{array}$ & $59.4(14.7)$ & $33.2(11.8)$ & $50.1(11.2)$ \\
\hline Osteoarthritis (hip) & 177 & Able to consent & Revisions, fractures & $67.6(11.2)$ & $24.4(6.6)$ & $49.4(12.5)$ \\
\hline Osteoarthritis (knee) & 189 & Able to consent & Revisions, fractures & $68.6(8.8)$ & 26.2 (7.9) & $50.9(12.4)$ \\
\hline CHF (usual vs. transitional care) & 191 & Speak English or French & Unable to consent & $75.7(9.9)$ & $29.9(8.2)$ & $51.0(9.6)$ \\
\hline CHF (partners in care) & 296 & Speak English or French & Unable to consent & $72.7(12.0)$ & $31.5(8.8)$ & $46.7(11.2)$ \\
\hline Chr. wound (uptake of evidence) & 117 & Speak English or French & Unable to consent & $74.1(12.5)$ & $32.3(9.9)$ & $48.8(10.7)$ \\
\hline Chr. wound (two models of care) & 211 & Speak English or French & Unable to consent & $68.4(13.9)$ & $35.7(9.8)$ & $49.1(11.2)$ \\
\hline Chr. wound (bandaging RCT) & 180 & Speak English or French & Unable to consent & $67.1(16.1)$ & $39.5(10.9)$ & $50.6(10.2)$ \\
\hline Chr. wound (new service delivery) & 652 & Speak English or French & Unable to consent & $72.2(13.7)$ & $31.8(9.6)$ & $47.9(12.0)$ \\
\hline Multiple sclerosis* & 276 & $\begin{array}{l}\text { Clinically definite MS } \\
\text { Communicate verbally }\end{array}$ & $\begin{array}{l}\text { Cognitive impairment } \\
\text { duration }>12 \text { months }\end{array}$ & $46.5(10.1)$ & 33.5 (10.6) & $46.0(12.2)$ \\
\hline
\end{tabular}

CHF: congestive heart failure;

Chr.: chronic;

SD: standard deviation;

PCS: physical component summary;

MCS: mental component summary

two studies), chronic wounds (leg ulcer, four studies) and MS. While HRQOL data do exist for these conditions, little of it is Canadian, and the opportunity to compare these five conditions is new, thus adding to the body of knowledge about the impact of chronic disease on HRQOL. These findings will also be of interest to those who provide care to patients with these conditions.

\section{Methods}

Details of the 10 studies are described below and are presented in Table 1. Data were collected at baseline through a combination of patient interview (SF-36 or SF-12, sociodemographic data) and chart review (clinical data). Ethics approval for each study was obtained from the Queen's University and Affiliated Teaching Hospitals Research Ethics Board or the Ottawa Health Research Institutes Ethics Board, as well as site-specific institutional reviews, where applicable. An application for the combined analysis was approved by the Queen's Research Ethics Board (approval number EPID-227-06).

\section{Measures}

The Medical Outcomes Trust 36-item health survey $(\mathrm{SF}-36)^{4}$ and its 12 -item subset, the SF-12,5 are among the most widely used instruments to measure
HRQOL. ${ }^{4,5}$ The SF-36 and SF-12 measure eight self-reported aspects of HRQOL, including physical function, physical role, bodily pain, general health, vitality, social function, emotional role and mental health. The Physical Component Summary (PCS) and Mental Component Summary (MCS) are standardized to a mean of 50, with a score above 50 representing better than average function and below 50 poorer than average function., ${ }^{5,6}$ Previous work by Ware et al. has noted a high degree of correspondence between the PCS and MCS obtained from the SF-36 and SF-12. Regression analyses to reproduce the PCS and MCS scores for the SF-36 using the SF-12 scores had $\mathrm{R}^{2}$ values in excess of 0.90 for both. ${ }^{5}$ In addition, an examination of the actual scores across 17 population and disease subgroup comparisons indicated that the average SF-36 and SF-12 PCS and MCS scores differed by less than one point, suggesting that the interpretation is the same and that comparisons are valid. ${ }^{5}$

\section{Databases and participants}

The renal failure database included all consenting patients receiving hemodialysis at Kingston General Hospital (KGH) and its affiliated satellite units in Kingston, Ontario. The SF-36 version 1.0 was administered at a routine hemodialysis visit. The hip and knee OA databases included all consenting primary elective total hip and total knee replacement patients on the waiting lists of five orthopedic surgeons in Kingston. The SF-36 version 1.0 was administered at the time of the six-week pre-surgical assessment.

The two CHF databases included all patients who had a diagnosis or exacerbation of CHF at hospital admission. Data for the first study (i.e. Usual Care versus Transition Care) were collected during hospitalization at two medical units of the Civic Campus at the Ottawa Hospital. Data for the second study (i.e. Partners in Care: CHF Study) were collected from patients recruited from 10 sites, including inpatient units, and community and specialty clinics in Ontario, New Brunswick, Manitoba and Illinois. HRQOL was assessed at the time of study entry using the SF-12 version 1.0. Although most were inpatients at the time of enrolment, they were seen early in their admission and the stays were typically brief. As most of the items on the SF-12 reference the past four weeks, the data are considered representative of the time when they were not hospitalized.

The chronic wound database (i.e. leg ulcers) was based on four studies, including the Prospective Study of the Uptake of EvidenceBased Guidelines in the Community; the 
Effectiveness and Efficiency of Two Models of Delivering Care to Chronic Wound Population; ${ }^{16}$ the Chronic Leg Ulcers in the Community Pre- and Post-Implementation of a New Service Delivery Model; ${ }^{17}$ and a Randomized Control Trial (RCT) of the Effectiveness of Two Compression Technologies. Patients were recruited from sites in Ontario (i.e. Ottawa, Kingston, Toronto, Hamilton, Niagara, KitchenerWaterloo and London), Manitoba (i.e. Winnipeg) and Saskatchewan (i.e. Regina and Saskatoon). SF-12 version 1.0 (version 2.0 for the RCT) data were collected as part of the baseline assessment.

The MS database included all consenting individuals with an appointment at the MS Clinic in Kingston over a one-year period. Two weeks before their appointment, patients received a package containing the SF-36 version 1.0 and a sociodemographic questionnaire. Those who consented returned the completed package at their appointment.

\section{Data management and statistical procedures}

All project databases were entered into SPSS (version 14.0 for Windows, Chicago, Illinois, 2005) for scoring and analysis. For the combined analysis, the variables contained in each, as well as the associated coding, were examined to find the common variables across the 10 databases. Key variables contained in each database were age, gender, whether the patient lived alone, cardiovascular disease, diabetes and "additional" comorbidities.

The definition for cardiovascular disease included hypertension, as this was important for the renal failure population. However, for the CHF group, it included cardiovascular disease other than congestive heart failure to avoid multicollinearity. While this resulted in a somewhat different adjustment for cardiovascular disease across the chronic conditions, it was felt that a crude adjustment was preferable to no adjustment at all. The diversity of the patient populations also resulted in the collection of different comorbidities. For example, very few comorbidities were collected for the MS sample, while a lengthy list was compiled for the heart failure studies.
As a result, only two comorbidities plus a category of "additional comorbidities" (i.e. defined simply as yes/no) could be drawn from each of the databases, and included comorbidities ranging from depression and sleep disorders to cancer, stroke and myocardial infarction. Variables such as education level, marital status, severity of disease and socio-economic status were not consistently collected across all databases. Patients under 25 years were excluded, as there were too few for comparison (i.e. two OA, four renal failure, three MS).

To facilitate comparison, age was categorized in 10-year increments as in the Canadian normative data for the SF-36. ${ }^{11}$ Once the 10 databases were collapsed into 5 condition-specific databases, the mean PCS and MCS scores were graphed by age group and condition, and compared to age- and sex-adjusted normative data. ${ }^{11}$ Linear regression models were developed for the PCS and the MCS, controlling for condition, age group, gender, living circumstances, cardiovascular disease, diabetes and additional comorbidities. All twoway interactions were also assessed. The condition with the highest mean age (i.e. CHF) was used as the reference condition, while the reference age group used was 25 to 34 years.

\section{Results}

\section{Response rates and demographics}

The 10 individual study sample sizes ranged from 117 to 652 participants, with a combined sample size of 2418 . The characteristics of patients in the five chronic conditions are displayed in Table 2. In all studies, the participation rates were high (i.e. $>77 \%$ ). For the renal failure database, 129 of 155 (83.2\%) provided consent, and age ranged from 25.5 to 89.8 years. For OA, 880 patients were eligible and 673 agreed to participate, for a response rate of $76.5 \%$. However, chart review was done after surgery and, consequently, only 366 participants had complete data, since 307 were still awaiting surgery at the study's end. Participant age ranged from 30.0 to 89.0 years, with a similar number of patients awaiting a total hip or knee replacement (i.e. 177 and 189, respectively).
The heart failure database contained 487 of 506 eligible patients (i.e. 96.2\%) who completed the HRQOL questionnaire. Patients ranged in age from 31.0 to 102.0 years. Four databases were represented in the chronic wound sample, and 1160 of 1470 (i.e. $78.9 \%$ ) patients completed the SF-12; ages for this sample ranged from 25.0 to 102.0 . For MS, 300 of 363 patients (i.e. $82.6 \%$ ) agreed to participate, with 276 completing the SF-36. This sample was the youngest, with ages ranging from 25.0 to 77.0 years, and only 10 individuals over 65 years. It also had far more women (i.e. 203) than men (i.e. 73), compared to the other studies where gender was more balanced.

\section{Descriptive statistics, physical component summary}

Figure 1 contains the graph of the mean values for the PCS for each age group by condition; the means and $95 \%$ confidence intervals are presented in the accompanying table. The differences between the normative data and the mean values of those with each of the chronic diseases were large, demonstrating a significant burden of illness. The renal failure group showed the greatest variation by age group, while the OA sample consistently had the lowest scores.

When examining all chronic diseases as a group ( $\mathrm{n}=2418)$, an initial decline levelled off as age increased. Starting with the 25 to 34 year age group and ending with the 75-plus age group, the mean values for the PCS were $41.4 \pm 10.1 ; 35.3 \pm$ $11.7 ; 32.3 \pm 10.3 ; 31.3 \pm 9.3 ; 30.6 \pm$ $9.6 ; 31.5 \pm 10.0$. The 10-point difference between the youngest (i.e. 41.4) and oldest (i.e. 31.5) groups was similar to the drop in the normative sample. Examining each disease group separately, this pattern was less clear, with some conditions (e.g. OA 55 to 64 and chronic wound 65 to 74) showing somewhat higher scores at older ages than in the adjacent, younger group. However, the confidence intervals were wide and overlapped for some conditions, suggesting that even though the differences were sometimes large, they were not necessarily statistically significant. 
FIGURE 1

Physical Component Summary scores by disease and age group

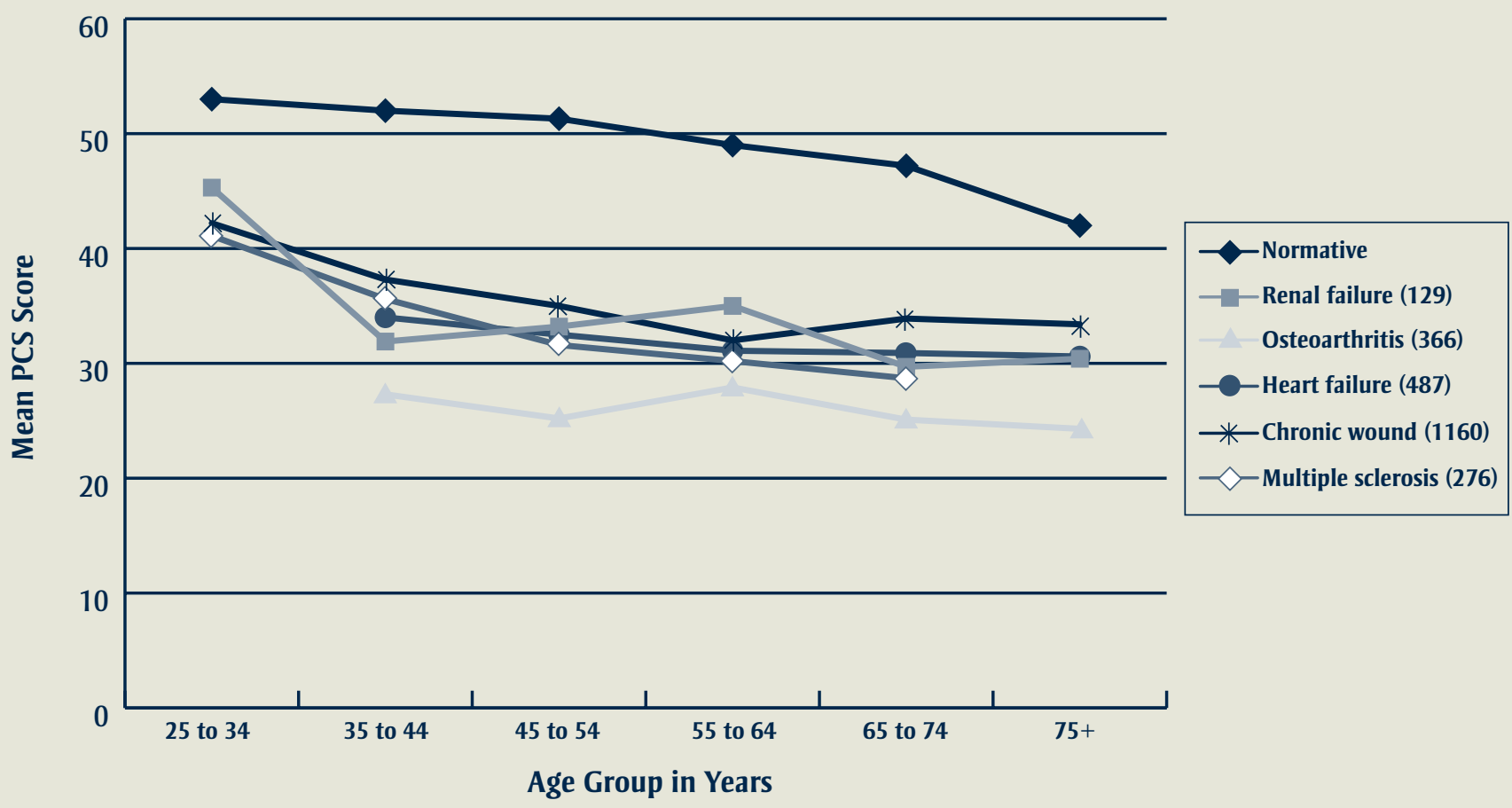

Footnote: Means and 95\% CIs by age group, physical component summary

\begin{tabular}{|c|c|c|c|c|c|c|}
\hline Sample (n) & 25 to 34 years & 35 to 44 years & 45 to 54 years & 55 to 64 years & 65 to 74 years & $75+$ years \\
\hline \multirow[t]{2}{*}{ Normative } & 53.0 & 52.0 & 51.3 & 49.0 & 47.2 & 42.0 \\
\hline & $52.2,53.7$ & $51.3,52.7$ & $50.9,51.7$ & $48.6,49.3$ & $46.8,47.6$ & $41.5,42.5$ \\
\hline Renal failure (129) & $36.8,53.7$ & $25.0,38.7$ & $27.0,39.3$ & $31.4,38.6$ & $26.1,33.2$ & $23.8,37.1$ \\
\hline \multirow[t]{2}{*}{ Osteoarthritis (366) } & $\mathrm{n} / \mathrm{a}$ & 27.3 & 25.2 & 27.9 & 25.1 & 24.3 \\
\hline & & $21.0,33.6$ & $23.1,27.3$ & $25.6,30.2$ & $24.1,26.2$ & $22.8,25.7$ \\
\hline Heart failure (487) & & $21.6,46.3$ & $28.8,36.2$ & $28.8,33.4$ & $29.4,32.3$ & 29.6, 31.6 \\
\hline \multirow[t]{2}{*}{ Chronic wound (1160) } & 42.2 & 37.3 & 35.0 & 32.0 & 33.9 & 33.4 \\
\hline & $37.2,47.3$ & $33.2,41.4$ & $33.1,36.9$ & $30.6,33.5$ & 32.7, 35.1 & $32.6,34.2$ \\
\hline \multirow[t]{2}{*}{ Multiple sclerosis (276) } & 41.1 & 35.6 & 31.6 & 30.2 & 28.7 & $\mathrm{n} / \mathrm{a}$ \\
\hline & $37.6,44.6$ & 33.4, 37.9 & $29.6,33.7$ & $27.7,32.8$ & $22.2,35.3$ & \\
\hline
\end{tabular}

Data were not graphed and $95 \% \mathrm{Cls}$ were not calculated when the sample size was $<5$. 
FIGURE 2

Mental component summary scores by disease and age group

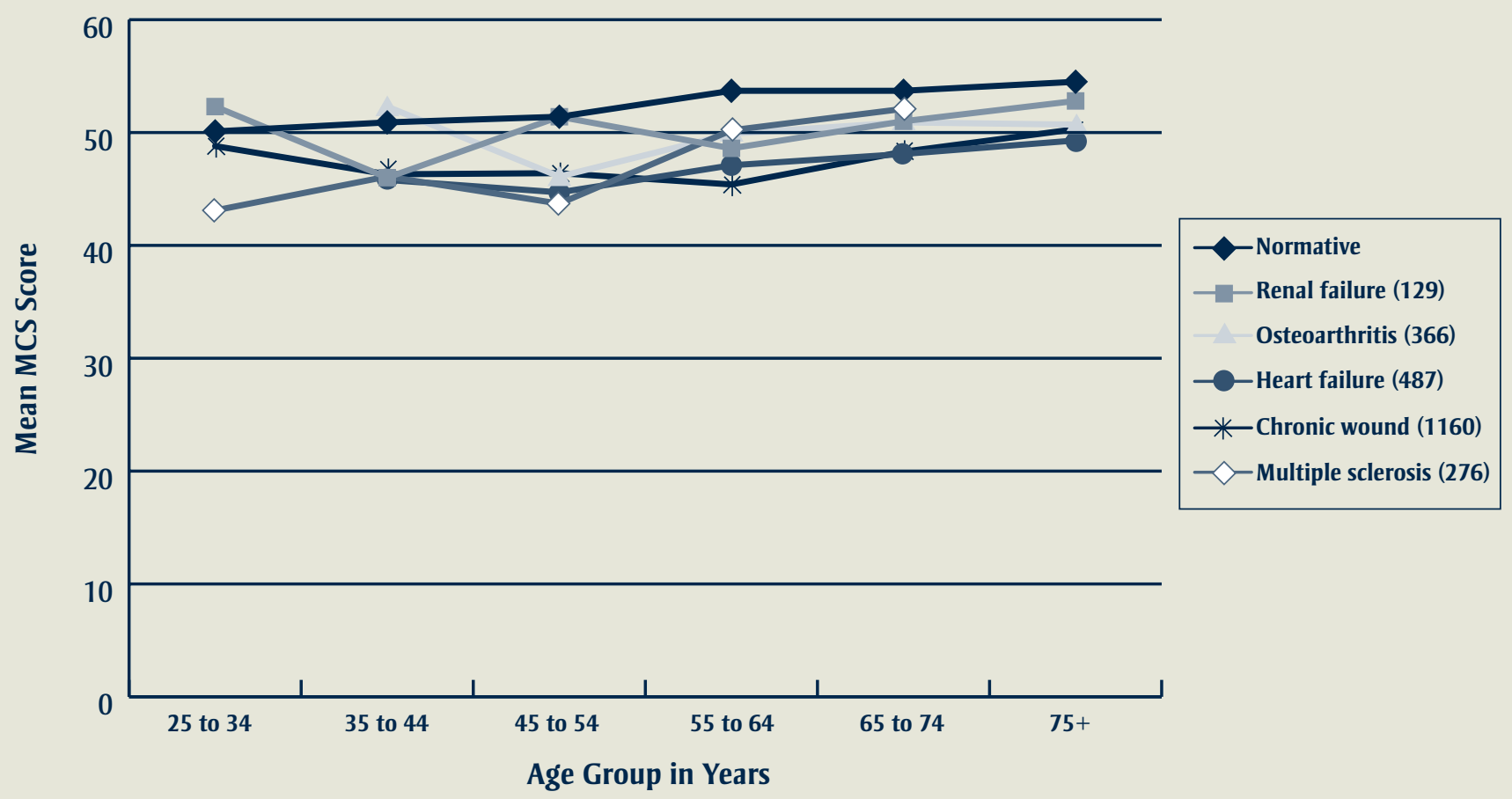

Footnote: Means and 95\% CIs by age group, physical component summary

\begin{tabular}{|c|c|c|c|c|c|c|}
\hline Sample (n) & 25 to 34 years & 35 to 44 years & 45 to 54 years & 55 to 64 years & 65 to 74 years & $75+$ years \\
\hline \multirow[t]{2}{*}{ Normative } & 50.1 & 50.9 & 51.4 & 53.7 & 53.7 & 54.5 \\
\hline & $49.2,51.1$ & $50.1,51.7$ & $51.0,51.8$ & $53.4,54.0$ & $53.4,54.0$ & $54.1,54.9$ \\
\hline \multirow[t]{2}{*}{ Renal failure (129) } & 52.3 & 46.0 & 51.4 & 48.6 & 51.0 & 52.8 \\
\hline & $45.5,59.0$ & $38.6,53.4$ & $47.1,55.6$ & $44.5,52.6$ & $46.8,55.2$ & $47.6,58.1$ \\
\hline \multirow[t]{2}{*}{ Osteoarthritis (366) } & $\mathrm{n} / \mathrm{a}$ & 52.3 & 46.1 & 49.9 & 50.9 & 50.7 \\
\hline & & $37.6,67.0$ & $42.4,49.8$ & $46.2,53.5$ & $49.0,52.7$ & $48.2,53.1$ \\
\hline \multirow[t]{2}{*}{ Heart failure (487) } & $\mathrm{n} / \mathrm{a}$ & 45.8 & 44.7 & 47.1 & 48.1 & 49.3 \\
\hline & & $34.7,56.8$ & $39.2,50.1$ & $44.1,50.1$ & $46.4,49.9$ & $47.9,50.6$ \\
\hline \multirow[t]{2}{*}{ Chronic wound (1160) } & 48.8 & 46.3 & 46.4 & 45.4 & 48.3 & 50.3 \\
\hline & $42.8,54.9$ & $42.5,50.2$ & $44.4,48.4$ & $43.3,47.5$ & $47.0,49.7$ & $49.4,51.2$ \\
\hline \multirow[t]{2}{*}{ Multiple sclerosis (276) } & 43.1 & 46.1 & 43.7 & 50.2 & 52.1 & $\mathrm{n} / \mathrm{a}$ \\
\hline & $38.6,47.7$ & $43.7,48.5$ & $41.1,46.4$ & $46.8,53.5$ & $41.7,62.4$ & \\
\hline
\end{tabular}

Data were not graphed and $95 \% \mathrm{Cls}$ were not calculated when the sample size was $<5$. 
TABLE 2

Sample characteristics for the five conditions

\begin{tabular}{|c|c|c|c|c|c|}
\hline Characteristic & Renal Failure $n=129$ & Osteoarthritis n = 366 & Heart Failure $n=487$ & $\begin{array}{l}\text { Chronic Wound } \\
\qquad n=1160\end{array}$ & $\begin{array}{l}\text { Multiple Sclerosis } \\
n=276\end{array}$ \\
\hline & n (\%) & n (\%) & n (\%) & n (\%) & n (\%) \\
\hline \multicolumn{6}{|l|}{ Age group } \\
\hline 25 to 34 years & $9(7.0)$ & $1(0.3)$ & $2(0.4)$ & $17(1.5)$ & $29(10.5)$ \\
\hline 35 to 44 years & $14(10.9)$ & $7(1.9)$ & $7(1.4)$ & $42(3.6)$ & $94(34.1)$ \\
\hline 45 to 54 years & $20(15.5)$ & $40(10.9)$ & $22(4.5)$ & $120(10.3)$ & $90(32.6)$ \\
\hline 55 to 64 years & 38 (29.5) & $56(15.3)$ & 58 (11.9) & 150 (12.9) & 53 (19.2) \\
\hline 65 to 74 years & $31(24.0)$ & 157 (42.9) & $136(27.9)$ & $267(23.0)$ & $8(2.9)$ \\
\hline $75+$ years & $17(13.2)$ & $105(28.7)$ & $262(53.8)$ & $564(48.6)$ & $2(0.7)$ \\
\hline Female & $54(41.9)$ & $202(55.2)$ & $236(48.5)$ & 649 (55.9) & $203(73.6)$ \\
\hline Live alone & $25(19.4)$ & 79 (21.6) & $195(51.5)$ & $420(36.2)$ & $35(12.7)$ \\
\hline \multicolumn{6}{|l|}{ Comorbidities } \\
\hline Cardiovascular* & $110(85.3)$ & $152(41.5)$ & $373(76.6) \neq$ & $701(60.4)$ & $47(17.0)$ \\
\hline Diabetes & 45 (34.9) & $13(3.6) \dagger$ & 158 (32.4) & 368 (31.7) & $12(4.3)$ \\
\hline \multirow[t]{2}{*}{ Additional } & $91(70.5)$ & 79 (21.6) & 435 (89.3) & $805(69.4)$ & $122(44.2)$ \\
\hline & Mean \pm SD & Mean \pm SD & Mean \pm SD & Mean \pm SD & Mean \pm SD \\
\hline Age & $59.4 \pm 14.7$ & $68.1 \pm 10.0$ & $73.9 \pm 11.3$ & $70.9 \pm 14.2$ & $46.5 \pm 10.1$ \\
\hline PCS & $33.2 \pm 11.8$ & $25.3 \pm 7.3$ & $30.9 \pm 8.6$ & $33.8 \pm 10.2$ & $33.5 \pm 10.6$ \\
\hline MCS & $50.1 \pm 11.2$ & $50.2 \pm 12.5$ & $48.4 \pm 10.9$ & $48.7 \pm 11.5$ & $46.0 \pm 12.2$ \\
\hline
\end{tabular}

SD: $\quad$ standard deviation;

PCS: physical component summary;

MCS: mental component summary

* Cardiovascular includes hypertension

† Insulin-dependent diabetes only for the OA group

$\ddagger$ Other than heart failure for the heart failure group

Descriptive statistics, mental component summary

Figure 2 contains the graph of the mean values for the MCS; the means and $95 \%$ confidence intervals are presented in the accompanying table. For those under 45 years, the sample sizes and resulting wide confidence intervals preclude valid comparisons for all but the MS sample aged 25 to 34 years, and both the MS and the chronic wound sample aged 35 to 44 years. In all three comparisons, those with the chronic disease scored substantially lower than the normative sample. The highest mean value for age groups over 55 years was in the normative sample, but the differences between the normative and condition-specific samples were small and, therefore, of questionable clinical significance. The MS sample had the lowest mean values for two age groups (i.e. 25 to 34 and 45 to 54 years), while the chronic wound sample had the lowest values for those in the 55 to 64 year age group. Several disease groups overlapped at the lowest value for the remaining three age groups.

When all samples were combined (i.e. $\mathrm{n}=$ 2418), mean MCS values were fairly stable for participants aged 25 to 54 years, after which there was a steady improvement by age category. Starting with the 25 to 34 year age group and ending with the 75-plus age group, the mean values for the MCS were $46.6 \pm 11.7 ; 46.4 \pm 11.9 ; 45.8 \pm 11.7 ; 47.4 \pm$ $12.7 ; 49.1 \pm 11.1 ; 50.1 \pm 11.2$. This increase in the older groups was similar to the normative sample. Within a disease, however, this pattern was less clear, with most diseases showing declines until the age group of 45 to 54 years. At this point, the mean MCS appeared to increase for all but the chronic wound sample, which increased after 55 years of age. However, as for the PCS results above, the confidence intervals were often wide and overlapping, and thus the data must be interpreted with caution.

Data were also grouped by gender within each disease to see if the pattern held true for both men and women. This was the case (data not shown); therefore, the results were not reported separately for men and women.

\section{Regression analyses}

Tables 3 and 4 contain the linear regression model for the PCS and MCS, respectively. Although all two-way interactions were tested, only two attained statistical significance in each model, all with negative coefficients (i.e. renal* additional comorbidities and chronic wound* additional comorbidities for the PCS; and 
TABLE 3

Linear regression model for the physical component summary

\begin{tabular}{|c|c|c|c|}
\hline Physical Component Summary $\left(r^{2}=0.14\right)$ & Coefficient & $95 \% \mathrm{Cl}$ & $p$-value \\
\hline Constant & 44.1 & $41.3,46.9$ & $<0.001$ \\
\hline \multicolumn{4}{|c|}{ Condition (reference $=$ heart failure $)(0=$ no, $1=$ yes $)$} \\
\hline Renal Failure & 0.9 & $-1.0,2.8$ & 0.367 \\
\hline Osteoarthritis & -8.6 & $-10.1,-7.1$ & $<0.001$ \\
\hline Chronic Wound & 1.9 & $0.9,3.0$ & $<0.001$ \\
\hline Multiple Sclerosis & -1.5 & $-3.2,0.3$ & 0.099 \\
\hline \multicolumn{4}{|l|}{ Age group (reference $=25$ to 34 years) } \\
\hline 35 to 44 years & -5.4 & $-8.2,-2.5$ & $<0.001$ \\
\hline 45 to 54 years & -7.7 & $-10.4,-5.0$ & $<0.001$ \\
\hline 55 to 64 years & -8.1 & $-10.8,-5.4$ & $<0.001$ \\
\hline 65 to 74 years & -7.9 & $-10.6,-5.2$ & $<0.001$ \\
\hline $75+$ years & -8.2 & $-10.9,-5.5$ & $<0.001$ \\
\hline Gender $(0=$ male $)$ & -1.3 & $-2.1,-0.5$ & 0.002 \\
\hline Cardiovascular Disease $(0=$ no $)$ & -1.1 & $-1.8,-0.4$ & 0.001 \\
\hline Diabetes $(0=$ no $)$ & -2.1 & $-3.1,-1.2$ & $<0.001$ \\
\hline Additional Comorbidities $(0=$ no $)$ & -3.5 & $-4.3,-2.6$ & $<0.001$ \\
\hline
\end{tabular}

Living circumstance was not significant $(p=0.52)$

$\mathrm{Cl}$ : confidence interval

TABLE 4

Linear Regression Model for the Mental Component Summary

\begin{tabular}{lccc}
\hline Mental Component Summary $\left(\mathbf{r}^{2}=\mathbf{0 . 0 5}\right)$ & Coefficient & $\mathbf{9 5 \%} \mathbf{C l}$ & $\boldsymbol{p}$-value \\
\hline Constant & 48.4 & $45.0,51.7$ & $<0.001$ \\
\hline Condition (reference = heart failure) $(0=$ no, $1=$ yes $)$ & & & \\
$\quad$ Renal Failure & 3.2 & $0.9,5.5$ & 0.006 \\
Osteoarthritis & 0.5 & $-1.3,2.3$ & 0.572 \\
Chronic Wound & 0.1 & $-1.1,1.4$ & 0.843 \\
Multiple Sclerosis & -0.3 & $-2.4,1.8$ & 0.755 \\
\hline Age group (reference $=25$ to 34 years) & & & \\
35 to 44 years & 0.3 & $-3.1,3.7$ & 0.861 \\
45 to 54 years & -0.1 & $-3.4,3.2$ & 0.963 \\
55 to 64 years & 2.1 & $-1.2,5.3$ & 0.218 \\
65 to 74 years & 3.9 & $0.7,7.1$ & 0.019 \\
$75+$ years & 5.3 & $2.1,8.5$ & 0.001 \\
\hline Gender $(0=$ male) & -1.3 & $-2.3,-0.4$ & 0.007 \\
Cardiovascular Disease $(0=$ no $)$ & -0.8 & $-1.7,-0.1$ & 0.042 \\
Diabetes $(0=$ no $)$ & -1.6 & $-2.7,-0.5$ & 0.006 \\
Additional Comorbidities $(0=$ no) & -2.6 & $-3.7,-1.5$ & $<0.001$ \\
\hline
\end{tabular}

Living circumstance was not significant $(p=0.85)$

$\mathrm{Cl}$ : confidence interval
$\mathrm{OA}^{*}$ additional comorbidities and MS* additional comorbidities for MCS). Given the limitations of the variable for additional comorbidities (described earlier), only the main effects were presented in the models.

The PCS model accounted for $14.4 \%$ of the variation in outcome. The OA sample scored significantly lower than the heart failure sample, while the chronic wound sample scored significantly higher than the heart failure sample. The difference between the MS and the heart failure sample approached significance. The renal failure sample did not differ significantly from the heart failure sample. All age groups scored significantly lower than the reference age group of 25 to 34 years. Men tended to have higher scores than women. Cardiovascular disease, diabetes and additional comorbidities were all associated with significantly lower mean PCS scores. Living circumstance was not a significant predictor of PCS.

The MCS model accounted for only 4.6\% of the variation in outcome. The similarity between the scores seen in Figure 2 was evident here as well, with only the renal failure sample scoring statistically significantly better than the heart failure sample. For the most part, the effect of increased age was positive and was statistically significant for age groups over 65 years. Women tended to score more poorly than men, as they did on the PCS. The effect of comorbidities was negative, although of borderline significance for cardiovascular disease. Living circumstances were not significantly associated with MCS.

\section{Discussion}

These data suggest a strong negative association between physical health status and both chronic disease and advanced age. However, mental health status remains relatively stable across disease groups and age groups. This phenomenon has been identified in other health conditions ${ }^{1,2,4,13-21}$ and the Canadian normative data, ${ }^{11}$ and is confirmed by the results of our analysis of five chronic conditions studied here.

The effect of advanced age on the PCS is strikingly negative, with effect sizes ranging from a five-point to an eight-point drop 
even after controlling for condition, gender and comorbidities. This is supported by the literature $\mathrm{e}^{2,4}$ and is not only statistically significant, but highly clinically relevant, given that a two- to three-point difference is likely to be clinically important. ${ }^{6}$ Only two other variables had a large effect, with the OA sample scoring an average of 8.6 points lower than the reference sample of heart failure; those with additional comorbidities also scored 3.5 points lower than those who did not have other comorbidities. However, it is likely that these estimates are conservative, given that the differences are relative to heart failure (i.e. used as reference group). Comparisons with a healthy population as reference category would likely show greater differences, but these data are only available in aggregate form.

Few variables had an effect size that exceeded two to three points on the MCS. Renal failure patients scored an average of 3.2 points higher than the reference category. Those with advanced age (i.e. 65 to 74 years and 75-plus years) scored higher (i.e. four points and five points, respectively) than the reference age group, which supports the literature on the effect of age on mental health. ${ }^{2,4,21}$ Additional comorbidities had a large negative association with the MCS, as those with additional comorbidities scored an average of 2.6 points lower than those without additional comorbidities. These findings are consistent with results from other studies that assessed the impact of the number of comorbidities on HRQOL. ${ }^{18-20,24,25}$

These results provide useful insights into the burden of illness experienced by persons with these chronic conditions. The finding that physical health status declines with increased age and disease burden is not new. ${ }^{1-3}$ However, these data provide useful estimates of the relative effect of age across five different diseases and confirm previous findings that identify declining physical function, but stable mental function, in those with chronic disease and/or increased age. These findings can also have important implications for the care and treatment of persons with these conditions. While it is not possible to predict when physical function is likely to decline for a specific case, the results can identify those at greater risk. In addition, evidence of better mental health in older age groups and in those whose illness has been diagnosed for some time may allow health care providers to focus in particular on the mental health of those recently diagnosed with a chronic disease. ${ }^{21}$ The finding that, on average, women had lower scores than men on both the PCS and the MCS suggests that they may be particularly vulnerable. Finally, the strong negative association between the comorbidities (i.e. cardiovascular, diabetes and "other comorbidities") and both mental and physical health status has been noted in other studies, ${ }^{18-20,24}$ suggesting that those with multiple comorbidities may be at greater risk for poor HRQOL outcomes.

The results should be interpreted within the limitations of the study. These data were obtained from 10 databases, with underlying study designs that varied in both purpose and methodology. As a result, only six variables were consistently collected across all databases; no consistent information was collected regarding illness severity, socio-economic status, education and social support, which are commonly associated with HRQOL. As a result, variables that are important determinants of physical (e.g. severity of illness) and/or mental health status (e.g. education and social support) could not be tested, thereby limiting our ability to develop the predictive models. Moreover, almost half (i.e. $48 \%$ ) of the subjects had chronic leg ulcers as the chronic disease, which limits the ability to generalize the findings.

In addition, one of the six variables (i.e. additional comorbidities) was based on the comorbidities collected within each study. Since some studies collected more than others, participants in those studies would be more likely to have a positive value for this variable. Moreover, there is increasing evidence that the severity as well as the number of comorbidities was an important consideration, ${ }^{19,20}$ and these data were not consistently collected within our studies. Future research would benefit from considering both factors, preferably with the use of a validated comorbidity index. Despite these limitations, this crude adjustment for illness severity seemed preferable to no adjustment.

Furthermore, sample sizes within the age groups for certain diseases were quite low. There were too few young patients with OA and heart failure, and too few older patients with MS to graph these age/disease groups. Even in cells with greater than five patients, some of the numbers were quite low. Consequently, large confidence intervals often overlapped, indicating that the results did not necessarily attain statistical significance even when the difference appeared large. Finally, our data are crosssectional and the age stratification is not the equivalent of a cohort that is followed over time.

Nevertheless, these data provide compelling evidence that, while physical function can be severely and negatively affected by both chronic disease and advanced age, mental health remains relatively high and stable, adding to the growing body of knowledge regarding the impact of increased age and chronic disease on HRQOL. Additional research with other disease groups, and longitudinal research in particular, will provide further insight into the complex relationship between chronic disease, physical health status, mental health status and advancing age.

\section{References}

1. Alonso J, Ferrer M, Gandek B, et al. Health-related quality of life associated with chronic conditions in eight countries: results from the international quality of life assessment (IQOLA) project. Qual Life Res. 2004;13:283-98.

2. Singer MA, Hopman WM, MacKenzie TA. Psychological adjustment in four chronic medical conditions. Qual Life Res. 1999; 8:687-91.

3. Brunet DG, Hopman WM, Singer MA, Edgar CM, MacKenzie TA. Measurement of health-related quality of life in multiple sclerosis patients. Can J Neurol Sci. 1996; 23:99-103. 
4. Ware JE, Snow KK, Kosinski M. SF-36 health survey: manual and interpretation guide. Boston (MA): The Health Institute, New England Medical Center; 1993.

5. Ware JE, Kosinski M, Keller SD. SF-12: how to score the SF-12 physical and mental health summary scales. Second ed. Boston (MA): The Health Institute, New England Medical Centre; 1995.

6. Ware JE, Kosinski M, Keller SD. SF-36 Physical and mental health summary scales: a user manual and interpretation guide. Boston (MA): The Health Institute, New England Medical Center; 1994.

7. Hobbs FD, Kenkre JE, Roalfe AK, Davis RC, Hare R, Davies MK. Impact of heart failure and left ventricular systolic dysfunction on quality of life: a cross-sectional study comparing common cardiac and medical disorders and a representative adult population. Eur Heart J. 2002;23:1867-76.

8. Jolly M. How does quality of life of patients with systemic lupus erythematosis compare with that of other common illnesses? J Rheumatol. 2005;32:706-8.

9. van der Wall JM, Terwee CB, van der Windt DA, Bouter LM, Dekker J. Health-related and overall quality of life of patients with chronic hip and knee complaints in general practice. Qual Life Res. 2005;14:95-803.

10. Salaffi F, Carotti M, Stancati A, Grassi W. Health-related quality of life in older adults with symptomatic hip and knee osteoarthritis: a comparison with matched healthy controls. Aging Clin Exp Res. 2005;17:255-63.

11. Hopman WM, Towheed T, Anastassiades T, et al. Canadian normative data for the SF-36 health survey. CMAJ. 2000;63:265-71.

12. Yost KJ, Haan MN, Levine RA, Gold EB. Comparing SF-36 scores across three groups of women with different health profiles. Qual Life Res. 2005;14:1251-61.
13. Kusek JW, Greene P, Wang SR, et al. Crosssectional study of health-related quality of life in African Americans with chronic renal insufficiency: the African American study of kidney disease and hypertension trial. Am J Kidney Dis. 2002;39:513-24

14. Groothoff JW, Grootenhuis MA, Offringa M, Gruppen MP, Korevaar JC, Heymans HSA. Quality of life in adults with end-stage renal disease since childhood is only partially impaired. Nephrol Dial Transplant. 2003;18:310-7.

15. Kaplan RM, Criqui MH, Denenberg JO, Bergan J, Fronek A. Quality of life in patients with chronic venous disease: San Diego population study. J Vasc Surg. 2003;37:1047-53.

16. Harrison MB, Browne GB, Roberts J, Tugwell P, Gafni A, Graham, ID. Quality of life of individuals with heart failure: a randomized trial of the effectiveness of two models of hospital-to-home transition. Med Care. 2002;40:271-82.

17. Harrison MB, Graham ID, Lorimer $\mathrm{K}$, Friedberg E, Pierscianowski T, Brandys T. Leg-ulcer care in the community, before and after implementation of an evidencebased service. CMAJ. 2005;172:1147-52.

18. Fortin M, Lapointe L, Hudon C, Vanasse A, Ntetu AL, Maltais D. Multimorbidity and quality of life in primary care : a systematic review. Health Qual Life Outcomes. 2004;2:51.

19. Fortin M, Bravo G, Hudon C, Lapointe L, Almirall J, Dubois MF, Vanasse A. Relationship between multimorbidity and health-related quality of life of patients in primary care. Qual Life Res. 2006;15(1):83-91.

20. Fortin M, Dubois MF, Hudon C, Soubhi H, Almirall J. Multimorbidity and quality of life: a closer look. Health Qual Life Outcomes. 2007;5:52.

21. Cassileth BR, Lusk EJ, Strouse TB, et al. Psychosocial status in chronic illness: a comparative analysis of six diagnostic groups. N Engl J Med. 1984;311: 506-11
22. Hopman WM, Berger C, Joseph L, et al. Stability of normative data for the SF-36: results of a three-year prospective study in middle-aged Canadians. Can J Public Health. 2004;95:387-91.

23. Hopman WM, Berger C, Joseph L, et al. The natural progression of health-related quality of life: results of a five-year prospective study of SF-36 scores in a normative population from the Canadian multicentre osteoporosis study (CaMos). Qual Life Res. 2006;15:527-36.

24. Bayliss EA, Bayliss MS, Ware JE Jr, Steiner JF. Predicting declines in physical function in persons with multiple chronic medical conditions: what we can learn from the medical problems list. Health Qual Life Outcomes. 2004;7(2):47.

25. Gadalla T. Association of comorbid mood disorders and chronic illness with disability and quality of life in Ontario, Canada. Chronic Dis Can. 2008;28(4):148-54. 
